\title{
Instrumentos de Avaliação das Funções Executivas: Revisão Sistemática dos Últimos Cinco Anos
}

\author{
Alanny Nunes de Santana ${ }^{1}$ \\ Universidade Federal de Pernambuco, Recife-PE, Brasil \\ Monilly Ramos Araujo Melo \\ Universidade Federal de Campina Grande, Campina Grande-PB, Brasil \\ Carla Alexandra da Silva Moita Minervino \\ Universidade Federal da Paraíba, João Pessoa-PB, Brasil
}

\section{RESUMO}

A avaliação das Funções Executivas (FE) torna-se problemática pela inexistência de um consenso metodológico quanto ao protocolo de avaliação. Destarte, foi realizada uma revisão sistemática objetivando verificar quais instrumentos foram utilizados nacional e internacionalmente nos últimos cinco anos para a avaliação das FE. Analisaram-se 35 artigos publicados em inglês e português nas bases SciELO, INDEXPSI, LILACS, PePSIC e nos periódicos CAPES. Observou-se, em sua maioria, estudos de tipo comparativo, correlacional e de validação/adaptação de instrumentos, respectivamente. Os instrumentos mais utilizados foram: Teste Wisconsin de Classificação de Cartas, Teste das Trilhas, Escalas Wechsler e os Testes de Stroop. Verificou-se a falta de instrumentos que avaliem os domínios das FE simultaneamente e a demanda pela construção de novos instrumentos, especialmente voltados para crianças e adolescentes.

Palavras-chave: função executiva; instrumentos; neuropsicologia.

ABSTRACT - Instruments for the Evaluation of Executive Functions: Systematic Review of the Previous Five Years The evaluation of executive functions (EF) is problematic due to the lack of methodological consensus regarding the evaluation protocol. Therefore, a systematic review was carried aiming to verify which instruments have been used nationally and internationally for the evaluation of EF in the previous five years. A total of 35 articles, published in English or Portuguese, in the SciELO, INDEXPSI, LILACS, PePSIC and CAPES periodicals databases were analyzed. Comparative studies were mainly found, followed by correlational and instrument validation/adaptation studies. The most used instruments were the Wisconsin Card Sorting Task, Trail Making Test, Wechsler Scales and Stroop Tests. A lack of instruments that evaluate the EF domains simultaneously was verified, as well as a need for the construction of new instruments, particularly directed toward children and adolescents.

Keywords: executive functions; neuropsychological instruments; neuropsychology.

RESUMEN - Instrumentos de Evaluación de Funciones Ejecutivas: Revisión Sistemática de los últimos Cinco años La evaluación de las Funciones Ejecutivas (FE) se vuelve problemática por la inexistencia de un consenso metodológico encuanto al protocolo de evaluación. Por lo tanto, se realizó una revisión sistemática com el objetivo de verificar qué instrumentos se utilizaron nacional e internacionalmente en los últimos cinco años para la evaluación de las FE. Se analizaron 35 artículos publicados en inglés y português en las bases SciELO, INDEXPSI, LILACS, PePSIC y en los periódicos CAPES. Se observó, en la mayoría, estudios de tipo comparativo, correlacional y de validación/adaptación de instrumentos, respectivamente. Los instrumentos más utilizados fueron: Prueba Wisconsin de clasificación de cartas, Test del Trazo, Escalas Wechsler y las Pruebas de Stroop. Se verifico la falta de instrumentos que evalúen los ámbitos de las FE simultáneamente y la demanda por la construcción de instrumentos, especialmente dirigidos para niños y adolescentes.

Palabras clave: funciónejecutiva; instrumentos; neuropsicología.

As Funções Executivas (FE) podem ser definidas enquanto um conjunto complexo e integrado de habilidades cognitivas que permitem ao indivíduo executar comportamentos direcionados a finalidades previamente estabelecidas (Kluwe-Schiavon, Viola, \& GrassiOliveira, 2012). São consideradas funções mentais superiores e responsáveis pela capacidade de autorregulação ou autogerenciamento, relacionando-se a vários componentes, a exemplo da atenção seletiva, controle inibitório, planejamento, organização, flexibilidade cognitiva e memória de trabalho ou memória operacional (Barros \& Hazin, 2013).

São variados os modelos teóricos que tratam das Funções Executivas, existindo, portanto, diversas 
definições e hipóteses relacionadas a essas habilidades, assim como distintas formas de avaliação (Kluwe-Schiavon et al., 2012). Diversos autores concordam ao mesmo tempo com a unicidade e a diversidade das FE, proposta por Miyake et al. (2000) (Diamond, 2013; Dias et al., 2015; Ferreira, Zanini, \& Seabra, 2015, Miyake et al., 2000). Assim, considera-se a existência de três componentes ou FE básicas, a saber: a memória de trabalho, a inibição ou controle inibitório e a flexibilidade cognitiva (Miyake et al., 2000).

Demonstra-se que um bom desempenho das FE implica que o indivíduo apresente domínios anatômicos e cognitivos categoriais intactos, em perfeita condição e funcionando de maneira adaptativa, o que por vezes não ocorre, gerando as chamadas disfunções executivas (Souza, Moll, Ignácio, \& Moll, 2008). Disfunções nas FE conduzem a prejuízos significativos que impactam o funcionamento pessoal do indivíduo em diferentes âmbitos, podendo-se mencionar as dificuldades quanto a capacidade de mudar mentalmente de foco, em manter a atenção, em aprender com os erros, habilidade de raciocínio pobre, dificuldade em planejar, bem como comportamento social e moral inadequados (Oliveira \& Nascimento, 2014).

Nesse sentido, a literatura na área das FE aponta, conforme a revisão realizada por Faria, Alves e Charchat-Fichman (2015), que diferentes instrumentos de avaliação neuropsicológica podem ser utilizados na avaliação das FE em adultos, destacando-se enquanto os de uso mais recorrente o Formulário de Teste de Trilha (TMT) B; o Teste de Fluência Verbal (VFT) - F, A e S; a Categoria de Animais de VFT; o Teste de Desenho do Relógio (CDT); os Subtítulos Digits Forward Backward (WAIS-R ou WAIS-III das Escalas Wechsler); o Teste de Stroop e o Teste de Classificação de Cartões de Wisconsin (WCST). Outra revisão também a respeito das FE, empreendida por Barros e Hazin (2013), revela, no que tange especificamente à avaliação das FE em crianças, que ocorreu um aumento da produção científica sobre o tema, considerando a importância dessas funções, bem como a relevância da avaliação precisa destas.

Barros e Hazin (2013) destacam, enquanto resultados da revisão realizada, que os principais instrumentos de avaliação das FE identificados foram o The Behavior Rating Inventory of Executive Function (BRIEF), as Escalas Wechsler e as tarefas baseadas nos paradigmas Go/No-Go e Stroop. Todavia, segundo os dados do estudo de Faria et al. (2015), demanda-se por pesquisas que ajudem a construir protocolos de avaliação das FE, considerando os diferentes níveis educacionais e as diferentes faixas etárias. Além disso, ressalta-se a importância da construção e utilização de instrumentos validados e adaptados para a avaliação dessas funções, nesse sentido, é relevante que na construção dessas ferramentas de avaliação sejam seguidas diretrizes, tais como as previstas no The Standards for
Educational and Psychological Testing (American Educational Research Association, American Psychological Association, \& National Council on Measurement in Education [AERA, APA, \& NCME], 2014). Nesse manual, encontram-se diretrizes relacionadas a fases de construção, validade, confiabilidade, direitos e responsabilidades dos responsáveis pelo teste, dentre outras.

Conforme Souza et al. (2008), as FE são as habilidades de mais complexa mensuração a partir de exames e testes, na medida em que estas geralmente se manifestam em ambientes característicos da vida real cotidiana que exigem condições de difícil reprodução em consultório. Além disso, a avaliação torna-se problemática pela inexistência de instrumentos padronizados únicos que meçam os domínios das FE simultaneamente, assim como de um consenso conceitual e metodológico quanto ao protocolo de avaliação (Nunes, Monteiro, \& Lopes, 2014; Zartman, Hilsabeck, Guarnaccia, \& Houtz, 2013). Enfatiza-se também que os testes são as principais ferramentas de mensuração dos déficits cognitivos provocados por lesões cerebrais ou disfunções cognitivas, não obstante, ocorre uma carência de instrumentos neuropsicológicos normatizados para a população brasileira (Fontoura, Rodrigues, Fonseca, Parente, \& Salles, 2011).

Diante da problemática apresentada, o presente artigo objetivou, a partir de uma revisão sistemática da literatura, verificar quais instrumentos são utilizados nacional e internacionalmente na avaliação das funções executivas nos últimos cinco anos. Objetiva-se também verificar as características dos desenhos metodológicos dos estudos empíricos que utilizam instrumentos na avaliação das FE, a frequência de publicações na área, os objetivos das pesquisas selecionadas, os parâmetros psicométricos dos instrumentos identificados, bem como averiguar as populações mais frequentemente investigadas nessas publicações. Justifica-se o presente estudo na medida em que a revisão sistemática sobre instrumentos de avaliação das FE mais recente data do ano de 2015 (Faria et al., 2015) e avalia apenas instrumentos voltados ao público adulto, não tendo sido encontrados artigos de revisão sobre instrumentos que apresentassem dados referentes a todas as faixas etárias e populações, revelando a relevância de uma revisão mais abrangente.

Destaca-se ainda a importância deste estudo na medida em que, segundo Carvalho et al. (2012), as FE estão entre os processos mentais mais investigados e discutidos atualmente, principalmente no que refere-se a avaliação destas, logo, pode-se fornecer um panorama geral da situação atual de pesquisas empíricas na área, bem como direcionar pesquisas futuras. Além disso, justifica-se, pois, segundo Verdejo-Garcia e Bechara (2010), a avaliação das FE interessa para além da clínica neuropsicológica, importando também a psicopatologia, a psicologia educacional, da saúde e ao estudo da adaptação dos mecanismos executivos frente às exigências ambientais. 


\section{Método}

No mês de setembro do ano de 2016, foi realizada uma busca nas bases de dados, Scientific Electronic Library Online (SciELO), Index Psi Periódicos (INDEXPSI), Literatura Latino-Americana e do Caribe em Ciências da Saúde (LILACS), Periódicos Eletrônicos de Psicologia (PePSIC) e nos periódicos disponíveis no site da Coordenação de Aperfeiçoamento de Pessoal de Nível Superior (CAPES), por artigos que tratassem de instrumentos de avaliação das funções executivas. Para tanto, objetivando a localização do maior número de artigos disponíveis, foram utilizadas as seguintes combinações de descritores/palavras-chave, a saber: funções and executivas and instrumentos and neuropsicologia, funções executivas and instrumentos and neuropsicologia, funções executivas and instrumentos, executive and functions and instruments and neuropsychology e executive functions and instruments.

Estudos coincidentes em duas ou mais fontes e/ ou entre a busca de diferentes palavras-chave foram contabilizados apenas uma vez. Artigos que não disponibilizaram texto completo e gratuitamente disponível, escritos em outro idioma (que não o inglês ou o português), resultantes de pesquisas não empíricas ou que não fazem referência direta ao tema foram excluídos da análise. Destaca-se, no que se refere aos estudos identificados, mas que não disponibilizaram o texto completo e gratuitamente disponível nas bases consultadas, que foram realizadas tentativas de contato via correio eletrônico com os autores, todavia, os estudos cujas tentativas de contato não foram respondidas foram excluídos.
Desse modo, incluiu-se na presente revisão apenas artigos que: 1 . apresentam referência direta ao tema "funções executivas e instrumentos neuropsicológicos"; 2. avaliam as FE a partir de instrumentos neuropsicológicos; 3. redigidos em português ou em inglês; 4. publicados entre os anos de 2010 e 2016; e 5. que apresentam resultados de pesquisas empíricas. Os artigos que se enquadraram nos requisitos apresentados anteriormente foram inicialmente selecionados a partir dos seus resumos. Os que se enquadraram mesmo após a análise dos resumos foram estudados em sua íntegra com ênfase na seção Método, considerando as recomendações do PRISMA (Galvão, Pansani, \& Harrad, 2015). A análise dos artigos consistiu nas seguintes etapas: 1 . verificar e analisar o desenho metodológico utilizado nos estudos (tipo de estudo, amostra, metodologia); 2. constatar e discutir brevemente sobre os parâmetros psicométricos dos instrumentos e baterias utilizadas na avaliação das FE; 3. verificar as populações investigadas; e 4. realizar a análise a partir de uma discussão sobre os resultados verificados em consonância com a literatura pertinente.

\section{Resultados e Discussão}

Encontraram-se, no total, 90 artigos, tendo sido excluídos um artigo não redigido em português ou inglês, 25 coincidentes/duplicados, nove que não faziam referência direta ao tema, 11 que não disponibilizavam texto completo e gratuitamente disponível nas referidas bases de dados e nove artigos não empíricos. Foram excluídos no total 55 artigos devido a não adequação aos critérios de inclusão, permanecendo para a análise 35 artigos, conforme o fluxograma abaixo:

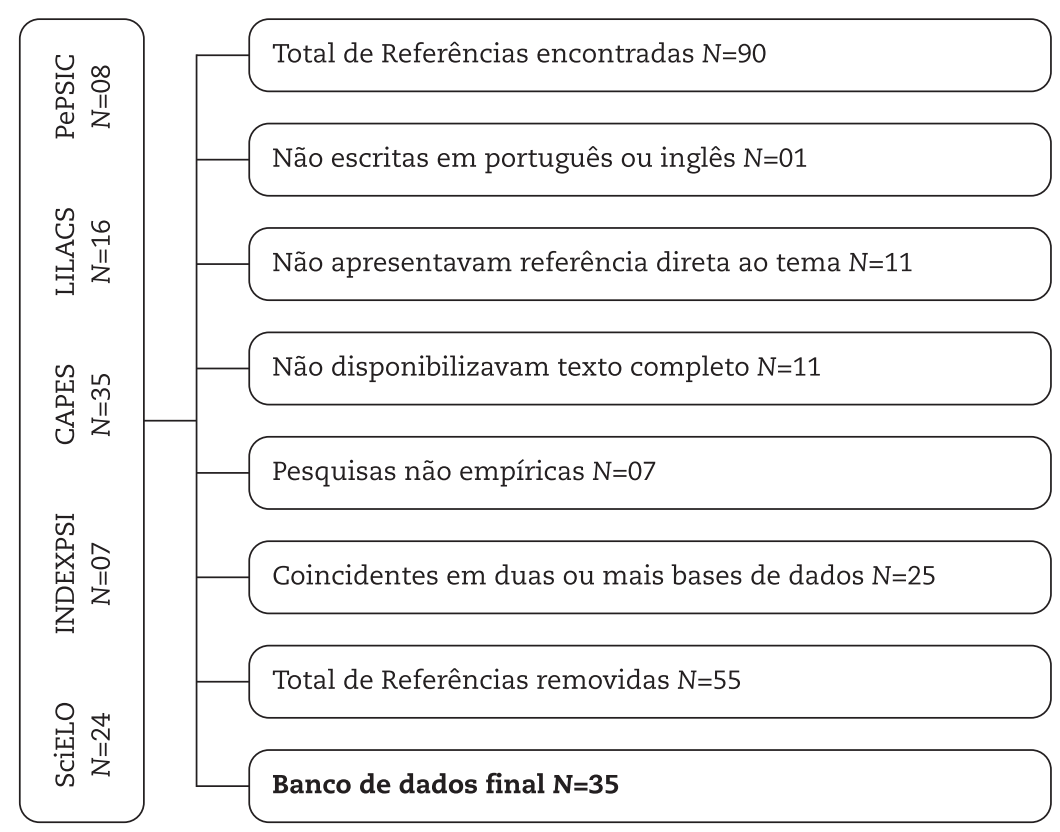


Constatou-se que 18 pesquisas apresentaram texto completo em inglês e 17 em português, demonstrando-se a prevalência dos textos escritos em inglês por apenas um artigo. A partir da busca realizada, observou-se um crescimento no número de pesquisas empíricas acerca dos instrumentos de avaliação neuropsicológica das funções executivas nos últimos anos, considerando o total de pesquisas publicadas no período de 2010 a 2012 (13 publicações) comparado com o período de 2013 a 2015 (22 publicações).

O crescimento no número de pesquisas empíricas acerca dos instrumentos de avaliação neuropsicológica das FE nos últimos três anos é um resultado que corrobora com a pesquisa realizada por Carvalho et al. (2012), na qual afirma-se que, com o avanço da neuropsicologia clínica, os estudos das funções executivas vêm aumentando de forma considerável nos últimos anos. Além disso, o aumento no número de pesquisas pode estar associado ao fato de que, conforme apontam Ramos e Hamdan (2016), os resultados obtidos por meio da avaliação neuropsicológica são o ponto de partida para o tratamento e planejamento de programas de reabilitação, destacando-se ainda a importância crescente dos estudos em neuropsicologia em diversos contextos, sejam de pesquisa, clínicos, forenses ou de educação. No entanto, confirma-se ainda, concordando com Barros e Hazin (2013), uma pequena quantidade de artigos publicados.

A análise do desenho metodológico dos artigos apontou para a prevalência de estudos de tipo comparativo (14) e correlacional (9). Além desses tipos de estudo, apresentaram-se também estudos de validação/adaptação de instrumentos (8), de construção de instrumentos (2) e estudos de caso (2), conforme a Tabela 1, que também apresenta os autores das publicações, os instrumentos utilizados para a avaliação das FE e as respectivas populações avaliadas nestas:

Tabela 1

Autores, Tipos de Estudo Verificados, Populações Avaliadas e Instrumentos

\begin{tabular}{|c|c|c|c|}
\hline Autores & Tipo de Estudo & População & Instrumentos \\
\hline $\begin{array}{l}\text { Balaguero, Mercé, Molina, } \\
\text { Tormos, e Rovira }\end{array}$ & $\begin{array}{c}\text { Estudo } \\
\text { comparativo }\end{array}$ & Adultos e Idosos & $\begin{array}{c}\text { Iowa Gambling Task (IGT) e } \\
\text { Balloon Analogue Risk Task (BART) }\end{array}$ \\
\hline $\begin{array}{l}\text { Bolshaw, Greca, Nardi, Júnior, } \\
\text { Fonseca, e Fernandez }\end{array}$ & $\begin{array}{l}\text { Estudo } \\
\text { comparativo }\end{array}$ & Adultos & $\begin{array}{c}\text { Teste das Trilhas, Teste de Stroop (cores } \\
\text { e palavras), NEUPSILIN, Teste de Hayling, } \\
\text { Escalas Wechsler e Bateria MAC }\end{array}$ \\
\hline $\begin{array}{l}\text { Boschloo, Krabbendam, } \\
\text { Aben, Groot, e Jolles }\end{array}$ & $\begin{array}{l}\text { Estudo } \\
\text { correlacional }\end{array}$ & Adolescentes & $\begin{array}{l}\text { The Behavior Rating Inventory of Executive } \\
\text { Function -Self-Report Version (BRIEF-SR) }\end{array}$ \\
\hline $\begin{array}{l}\text { Brito, Areosa, Lopes, } \\
\text { e Argimon. }\end{array}$ & $\begin{array}{c}\text { Estudo } \\
\text { correlacional }\end{array}$ & Idosos & WCST \\
\hline $\begin{array}{l}\text { Cardoso, Zimmermann, Paraná, } \\
\text { Gindri, Pereira, e Fonseca }\end{array}$ & $\begin{array}{l}\text { Validação de } \\
\text { instrumento }\end{array}$ & Adultos & Tarefa do Hotel \\
\hline $\begin{array}{l}\text { Carvalho, Cardoso, Cotrena, } \\
\text { Bakos, Kristensen, e Fonseca }\end{array}$ & $\begin{array}{l}\text { Estudo } \\
\text { correlacional }\end{array}$ & Adultos & $\begin{array}{l}\text { Teste das Trilhas, Teste de Hayling, } \\
\text { Iowa Gambling Task (IGT) e WCST }\end{array}$ \\
\hline $\begin{array}{c}\text { Coelho, Albuquerque, } \\
\text { e Simões }\end{array}$ & $\begin{array}{c}\text { Estudo } \\
\text { comparativo }\end{array}$ & $\begin{array}{c}\text { Crianças e } \\
\text { Adolescentes }\end{array}$ & $\begin{array}{c}\text { Bateria BANC, Figuras Complexas de Rey, } \\
\text { Torre de Coimbra e Teste das Trilhas }\end{array}$ \\
\hline $\begin{array}{c}\text { Duchesne, Mattos, Appolinário, } \\
\text { Freitas, Coutinho, Santos, } \\
\text { e Coutinho }\end{array}$ & $\begin{array}{l}\text { Estudo } \\
\text { comparativo }\end{array}$ & Adultos & $\begin{array}{c}\text { Teste das Trilhas, Teste de Stroop, WCST, } \\
\text { Escalas Weschler e Behavioural Assessment } \\
\text { of Dysexecutive Syndrome (BADS) }\end{array}$ \\
\hline Ferreira, Zanini, e Seabra & $\begin{array}{c}\text { Estudo } \\
\text { correlacional }\end{array}$ & $\begin{array}{c}\text { Crianças e } \\
\text { Adolescentes }\end{array}$ & Torre de Hanói e Escalas Wechsler \\
\hline $\begin{array}{c}\text { Fonseca, Lima, Ims, Coelho, } \\
\text { e Ciasca }\end{array}$ & $\begin{array}{c}\text { Estudo } \\
\text { correlacional }\end{array}$ & Crianças & $\begin{array}{l}\text { Teste das Trilhas, Teste de Stroop e } \\
\text { Torre de Londres }\end{array}$ \\
\hline $\begin{array}{c}\text { Fonseca, Zimmermann, } \\
\text { Cotrena, Cardoso, Kristensen, } \\
\text { e Grassi-Oliveira }\end{array}$ & $\begin{array}{l}\text { Estudo } \\
\text { comparativo }\end{array}$ & Adultos e Idosos & $\begin{array}{l}\text { Teste das Trilhas, Teste de Hayling e } \\
\text { Iowa Gambling Task (IGT) }\end{array}$ \\
\hline $\begin{array}{l}\text { Fontoura, Rodrigues, Jaqueline, } \\
\text { Fonseca, Parente, e Salles }\end{array}$ & $\begin{array}{l}\text { Validação de } \\
\text { instrumento }\end{array}$ & $\begin{array}{l}\text { Adolescentes, } \\
\text { Adultos e Idosos }\end{array}$ & NEUPSILIN-Af \\
\hline $\begin{array}{c}\text { Freire, Gonçalves, Moretti, } \\
\text { Tabaquim, e Crenitte }\end{array}$ & Estudo de caso & Adolescente & WCSTe Escalas Wechsler \\
\hline $\begin{array}{l}\text { Hovik, Plessen, Cavanna, } \\
\text { Skogli, Andersen, e Øie }\end{array}$ & $\begin{array}{l}\text { Estudo } \\
\text { comparativo }\end{array}$ & Crianças & $\begin{array}{c}\text { Teste de Stroop, Behavior Rating } \\
\text { Inventory of Executive Function (BRIEF), } \\
\text { Letter-Number Sequencing Test (LNS) e } \\
\text { Iowa Gambling Task (IGT) }\end{array}$ \\
\hline $\begin{array}{l}\text { Ihnen, Antivilo, Muñhoz-Neira, } \\
\text { e Slachevsky }\end{array}$ & $\begin{array}{l}\text { Validação de } \\
\text { instrumento }\end{array}$ & Adultos e Idosos & $\begin{array}{l}\text { Ineco Frontal Screening (IFS), WCST e } \\
\text { Addenbrooke's Cognitive Examination }\end{array}$ \\
\hline
\end{tabular}


Tabela 1 (continuação)

Autores, Tipos de Estudo Verificados, Populações Avaliadas e Instrumentos

\begin{tabular}{|c|c|c|c|}
\hline Autores & Tipo de Estudo & População & Instrumentos \\
\hline Lima, Azoni, e Ciasca & $\begin{array}{c}\text { Estudo } \\
\text { comparativo }\end{array}$ & Crianças & WCST e Escalas Wechsler \\
\hline $\begin{array}{l}\text { Lopes, Nascimento, Esteves, } \\
\text { Iatchac, e Lima }\end{array}$ & $\begin{array}{c}\text { Estudo } \\
\text { comparativo }\end{array}$ & Idosos & WCST e Escalas Wechsler \\
\hline $\begin{array}{c}\text { Lopes, Nascimento, Esteves, } \\
\text { Terroso, e Argimon }\end{array}$ & $\begin{array}{c}\text { Estudo } \\
\text { comparativo }\end{array}$ & Idosos & WCST e Escalas Wechsler \\
\hline Lopes & $\begin{array}{c}\text { Estudo } \\
\text { comparativo }\end{array}$ & Idosos & WCST e Escalas Wechsler \\
\hline Martino, Strejilevich, e Manes & $\begin{array}{c}\text { Estudo } \\
\text { comparativo }\end{array}$ & Idosos & WCST e Escalas Wechsler \\
\hline $\begin{array}{l}\text { Mata, Sallum, Moraes, Miranda, } \\
\text { e Malloy-Diniz }\end{array}$ & $\begin{array}{l}\text { Construção de } \\
\text { instrumento }\end{array}$ & Crianças & Children's Gambling Task (CGT) \\
\hline $\begin{array}{c}\text { Möller, Boussard, Oldenburg, } \\
\text { e Bartfai }\end{array}$ & $\begin{array}{l}\text { Estudo } \\
\text { correlacional }\end{array}$ & Adultos & Escalas Wechsler e Teste Stroop \\
\hline $\begin{array}{l}\text { Morrison, Giles, Ryan, Baum, } \\
\text { Dromerick, Polatajko, e Edwards }\end{array}$ & $\begin{array}{c}\text { Estudo } \\
\text { comparativo }\end{array}$ & Adultos e Idosos & $\begin{array}{l}\text { Multiple Errands Test-Revised (MET-R) e } \\
\text { Executive Function Performance Test (EFPT) }\end{array}$ \\
\hline $\begin{array}{l}\text { Netto, Greca, Ferracini, Pereira, } \\
\text { Bizzo, Doring, Kubo, Bahia, } \\
\text { Fonseca, e Gasparetto }\end{array}$ & $\begin{array}{l}\text { Estudo } \\
\text { correlacional }\end{array}$ & Adultos e Idosos & $\begin{array}{c}\text { Teste de Trilhas, Teste de Stroop, WCST, } \\
\text { Teste de Hayling, Bateria Montreal de } \\
\text { Avaliação da Comunicação (MAC) e } \\
\text { Escalas Wechsler }\end{array}$ \\
\hline Nunes, Monteiro, e Lopes & $\begin{array}{l}\text { Estudo } \\
\text { comparativo }\end{array}$ & Adultos & $\begin{array}{c}\text { Ineco Frontal Screening (IFS) e Behavioural } \\
\text { Assessment of Dysexecutive Syndrome } \\
\text { (BADS) }\end{array}$ \\
\hline Oliveira e Nascimento & $\begin{array}{l}\text { Construção de } \\
\text { instrumento }\end{array}$ & Adultos e Idosos & Escala de Autorrelato \\
\hline $\begin{array}{l}\text { Oliveira, Pedron, Gurge, } \\
\text { Reppold, e Fonseca }\end{array}$ & $\begin{array}{c}\text { Estudo } \\
\text { comparativo }\end{array}$ & Adultos & Teste de Hayling e Teste das Trilhas, \\
\hline $\begin{array}{c}\text { Pavan, Casarin, Pagliarin, } \\
\text { e Fonseca }\end{array}$ & Estudo de caso & Adulto & $\begin{array}{l}\text { WCST, Teste de Trilhas e Escalas } \\
\text { Wechsler }\end{array}$ \\
\hline Pazeto, Seabra, e Dias & $\begin{array}{c}\text { Estudo } \\
\text { correlacional } \\
\end{array}$ & Crianças & Teste de Stroop e Testes das Trilhas, \\
\hline $\begin{array}{l}\text { Pedron, Ferrão, Gurgel, } \\
\text { e Reppold }\end{array}$ & $\begin{array}{l}\text { Estudo } \\
\text { correlacional }\end{array}$ & Adultos e Idosos & $\begin{array}{c}\text { Teste de Stroop, Figuras Complexas } \\
\text { de Rey, WCST, Go No-Go Task e Escalas } \\
\text { Wechsler }\end{array}$ \\
\hline Ritter, Perrig, Steinlin, e Everts & $\begin{array}{l}\text { Estudo } \\
\text { comparativo }\end{array}$ & Crianças & $\begin{array}{l}\text { Teste das Trilhas, Delis-Kaplan Executive } \\
\text { Function System (D-KEFS), Escalas } \\
\text { Wechsler e Behavior Rating Inventory of } \\
\text { Executive Function (BRIEF) }\end{array}$ \\
\hline $\begin{array}{c}\text { Salles, Fonseca, Cruz-Rodrigues, } \\
\text { Melo, Barbosa, e Miranda }\end{array}$ & $\begin{array}{l}\text { Validação de } \\
\text { instrumento }\end{array}$ & Crianças & NEUPSILIN-INF \\
\hline $\begin{array}{c}\text { Silva, Zongo, Madureira, } \\
\text { e Peixoto }\end{array}$ & $\begin{array}{l}\text { Validação de } \\
\text { instrumento }\end{array}$ & Adultos & Ineco Frontal Screening (IFS) \\
\hline Wachholz e Yassuda & $\begin{array}{c}\text { Estudo } \\
\text { comparativo }\end{array}$ & Idosos & $\begin{array}{c}\text { Bateria Breve de Rastreio Cognitivo } \\
\text { (BBRC) e Escalas Weschler }\end{array}$ \\
\hline $\begin{array}{l}\text { Zartman, Hilsabeck, } \\
\text { Guarnaccia, e Houtz }\end{array}$ & $\begin{array}{l}\text { Validação de } \\
\text { instrumento }\end{array}$ & Idosos & $\begin{array}{l}\text { Pillbox Test, Teste de Stroop, Figuras } \\
\text { Complexas de Rey, Teste das Trilhas, } \\
\text { Teste WCST e Escalas Wechsler }\end{array}$ \\
\hline
\end{tabular}

Verificou-se nos estudos de tipo comparativo e de tipo correlacional que os participantes foram confrontados levando-se em consideração os escores em instrumentos de avaliação neuropsicológica das FE, escores estes analisados a partir de procedimentos de estatística descritiva. Nos estudos de validação/adaptação de instrumentos, foi possível verificar a utilização de variadas técnicas, sejam estas de validade discriminante (MANOVA, curva de ROC), validade convergente e divergente (correlações de Pearson e Spearman), evidência de confiabilidade (coeficiente alfa de Cronbach), validade de conteúdo (análise por juízes/experts, testes pilotos), análise da variância (qui-quadrado) e comparação das Médias (Testes Kruskall-Wallis e Mann-Whitney). 
Destaca-se que os processos de validação e adaptação de instrumentos, mencionados nos artigos analisados, são importantes para que o profissional sinta-se seguro quanto à eficácia do método utilizado.

Afirma-se que os testes psicológicos como instrumentos de medida devem apresentar certas características que justifiquem poder confiar nos dados que produzem, sendo essas características a validade e a precisão/ fidedignidade (Pasquali, 2001). Em âmbito internacional, Ottati e Noronha (2003) enfatizam que a preocupação com a construção e o uso de instrumentos psicológicos é também uma realidade, tendo sido criadas comissões de testes, objetivando a promoção e a potencialização do uso adequado dos instrumentos psicológicos. Nos Estados Unidos, por exemplo, existem publicações anuais que oferecem importantes informações sobre os testes, incluindo levantamentos de tipos de instrumentos e a verificação de estudos de validade e precisão, tais como o Mental Measurements Year books e o Test Critique do Buros Institute of Mental Measurements.

Nos artigos que versavam sobre a adaptação de instrumentos, foram observados o uso de técnicas, como: revisão da literatura pertinente, tradução do instrumento original, análise do instrumento original, análise de juízes especialistas na área da saúde, análise de juízes especialistas em neuropsicologia, desenvolvimento de novos estímulos e estudos-piloto (Cardoso et al., 2015; Fontoura et al., 2011; Salles et al., 2011). Afirma-se que o processo de adaptação refere-se a uma tarefa complexa que exige comprovar tanto as evidências acerca da equivalência semântica dos itens, quanto as evidências psicométricas da nova versão do instrumento, levando em consideração a adequação cultural, ou seja, o preparo do instrumento para seu uso em outro contexto (Borsa, Damásio, \& Bandeira, 2012).

Nos processos de construção de instrumentos (2), percebeu-se o uso de metodologias distintas. Na construção do Children's Gambling Task (Mata, Sallum, Moraes, Miranda, \& Malloy-Diniz, 2013), foram consideradas as técnicas de validade convergente, por meio da correlação entre o desempenho no Columbia Mental Maturity Scale (CMMS) e no Children's Gambling Task; e a validade diferencial, a partir da análise de variância (ANOVA) que foi utilizada para as variáveis Idade e Sexo. Enquanto isso, na construção da Escala para Avaliação do Planejamento Cognitivo, abordou-se os polos teórico, empírico e analítico por meio dos seguintes passos: revisão teórica, entrevista com a população meta, construção de itens/ instrumento, análise de juízes, análise semântica, caracterização da amostra, aplicação do estudo-piloto, análise psicométrica (conduzida segundo a Teoria Clássica dos Testes), análise textual e aplicação da escala (Oliveira \& Nascimento, 2014).

Destaca-se que apenas um dos artigos tratava-se do processo de construção e desenvolvimento de um instrumento computadorizado (Computerized Adaptive
Test - CAT), sendo este o teste Gambling para crianças (Mata et al., 2013). Nesse ínterim, nas últimas quatro décadas tem havido um crescimento na elaboração e uso dos testes computadorizados como uma alternativa viável aos testes de papel e lápis, já que o CAT apresenta diversas vantagens, sendo estas relacionadas a administração e correção do teste, precisão das estimativas, segurança do teste, motivação e tecnologia, além de viabilizarem a avaliação por múltiplos estímulos com maior precisão e menor tempo. Contudo, a transição dos testes tradicionais para os CATs é de alto custo e demanda conhecimentos interdisciplinares, como de tecnologia da informação, já que estes requerem mais recursos financeiros e humanos do que a versão papel-e-lápis, de modo que o seu desenvolvimento ainda demonstra-se incipiente (Luecht \& Sireci, 2011).

Os resultados da análise do desenho metodológico ainda apontam o estudo de caso como procedimento de pesquisa científica das FE, apresentando-se em dois artigos. Um deles objetivou determinar as alterações neuropsicológicas em um indivíduo adulto que sofreu um acidente vascular cerebral (AVC), utilizando para tal avaliação a Bateria Montreal de Avaliação da Comunicação, o Teste de trilhas, as Escalas Wechsler e o WCST (Pavan, Casarin, Pagliarin, \& Fonseca, 2015). O segundo estudo de caso analisou um adolescente a partir dos instrumentos Escalas Wechsler e WCST no intuito de verificar seu desempenho na aprendizagem, considerando-se que este apresenta a Síndrome Velocardiofacial (Freire, Gonçalves, Moretti, Tabaquim, \& Crenitte, 2015). O estudo de caso mostra-se, portanto, como uma importante ferramenta que permite um profundo estudo de um objeto, sendo um meio para conseguir a avaliação mais completa e singular possível. No entanto, tal ferramenta metodológica pode levar a certa falta de rigor, demandando muito tempo e não gerando margem para generalizações (Goode \& Hatt, 1973).

Especificamente no que se refere aos instrumentos para avaliação das FE, foi possível verificar que, dentre os utilizados pelos autores, 24 apresentaram validade e adaptação à realidade nacional, enquanto que sete foram utilizados apenas nos estudos internacionais (INECO frontal Screening, Design Fluency Test, Multiple Errands Test - revised, Balloon Analogue Risk Task, Letter - Number Sequencing Test, Pillbox Test, Controlled oral Word Association Test e a Bateria de Avaliação Neuropsicológica de Coimbra). Destaca-se que se verificou a prevalência de instrumentos que não avaliam apenas as funções executivas, examinando também outras habilidades cognitivas, a exemplo das Escalas Wechsler, a Bateria breve de rastreio cognitivo, Addenbrooke's Cognitive Examination, o NEUPSILIN e o Frontal Assessment Battery.

Os estudos encontrados empregaram uma grande variedade de instrumentos e baterias de avaliação neuropsicológica, objetivando avaliar as diferentes habilidades executivas. Conforme Barros e Hazin (2013), a 
ampla diversidade de instrumentos utilizados pode ser justificada pelos múltiplos componentes investigados, sendo ainda um reflexo da falta de concordância metodológica dessa temática e das diferentes correntes teóricas existentes no estudo dessas habilidades. Os instrumentos/baterias formais de mensuração das FE utilizados nos artigos incluídos, bem como a frequência de utilização encontram-se dispostos na Tabela 2.

Tabela 2

Instrumentos Utilizados para a Avaliação e Frequência

\begin{tabular}{|c|c|}
\hline Instrumento & Frequência \\
\hline Teste Wisconsin de Classificação de Cartas (WCST) & 14 \\
\hline Escalas Wechsler (WISC/WAIS/WIAT e WPPSI) & 14 \\
\hline Teste das Trilhas (Trail Making Test) & 13 \\
\hline Teste Stroop (numérico, sonoro e pictórico) & 10 \\
\hline Hayling Test & 5 \\
\hline Iowa Gambling Task (IGT) & 5 \\
\hline Teste das Torres (Londres e Hanói) & 5 \\
\hline The Behavior Rating Inventory of Executive Function (BRIEF) & 3 \\
\hline Instrumento de Avaliação Neuropsicológica Breve (NEUPSILIN) & 3 \\
\hline Figura Complexa de Rey & 3 \\
\hline Verbal Fluency Tasks (Teste de fluência verbal) & 3 \\
\hline INECO Frontal Screening (IFS) & 3 \\
\hline Testes de Cancelamento (Cancellation) & 3 \\
\hline Bateria Breve de Rastreio Cognitivo (BBRC) & 2 \\
\hline Bateria Montreal de Avaliação da Comunicação (MAC) & 2 \\
\hline Paradigma Go/No-Go & 2 \\
\hline Clock Drawing Test (CDT) & 2 \\
\hline Behavioural Assessment of Dysexecutive Syndrome (BADS) & 2 \\
\hline Frontal Assessment Battery (FAB) & 1 \\
\hline Design Fluency Test (DFT) & 1 \\
\hline Multiple Errands Test-Revised (MET-R) & 1 \\
\hline Escala de Autorrelato & 1 \\
\hline Balloon Analogue Risk Task (BART) & 1 \\
\hline Tarefa do Hotel & 1 \\
\hline Letter-Number Sequencing Test (LNS) & 1 \\
\hline Pillbox Test & 1 \\
\hline Controlled Oral Word Association Test (COWAT) & 1 \\
\hline Addenbrooke’s Cognitive Examination & 1 \\
\hline Bateria de Avaliação Neuropsicológica de Coimbra (BANC) & 1 \\
\hline Delis-Kaplan Executive Function System (D-KEFS) & 1 \\
\hline Executive Function Performance Test (EFPT) & 1 \\
\hline
\end{tabular}

Os dois instrumentos mais utilizados pelos autores foram o WCST (Cunha, 2005) e o Teste das Trilhas (Ait, 1944; Magila, \& Caramelli, 2000; Spreen, \& Strauss, 1991). O mais aplicado ao longo das pesquisas foi o WCST, verificado em 14 dos artigos analisados. Corrobora-se, portanto, com Silva-Filho, Pasian e Barboza (2013) ao afirmarem que o WCST é o instrumento considerado padrão ouro para avaliar as FE, sendo um dos mais utilizados na avaliação do domínio flexibilidade mental na resolução de problemas, atenção e impulsividade. A alta frequência da sua utilização pode relacionar-se ao fato de que já encontra-se adaptado e padronizado a realidade brasileira e que seu manual contém tabelas normativas para todas as idades, auxiliando a transformação dos escores brutos em percentis, escore T e escore padrão, de modo a facilitar sua aplicação em populações das mais diferentes faixas etárias, bem como a utilização dos dados em pesquisas (Heaton, Chelune, Talley, Kay, \& Curtiss, 2004).

O segundo instrumento mais utilizado foi o Teste das Trilhas (Trail Making Test), apresentando-se em 13, dos 35 artigos analisados. O teste examina a coordenação visuomotora, a velocidade de processamento, a atenção concentrada, a atenção alternada, a flexibilidade cognitiva 
e a inibição (Netto et al., 2011; Pavan et al., 2015). Sua aplicação na avaliação das FE pode justificar-se pelo fato deste aferir a inibição e a flexibilidade cognitiva, dois dos três componentes básicos das FE, conforme Miyake et al. (2000), e também por ser de fácil aplicação e baixo custo.

É possível verificar que, além do uso de testes específicos, também são utilizadas pelos autores/pesquisadores algumas baterias, destacando-se as escalas Wechsler (WISC/WAIS/WIAT e WPPSI), utilizadas em 14 artigos; a Bateria Breve de Rastreio Cognitivo (BBRC), as tarefas do Paradigma Go/No-Go e a Bateria Montreal de avaliação da comunicação (MAC), todas utilizadas em dois artigos, dos 35 analisados. Além das baterias foi também utilizada a escala The Behavior Rating Inventory of Executive Function (BRIEF), presente em três artigos.

Nota-se que a grande maioria das pesquisas analisadas (30 dos 35 estudos) utilizou mais de um instrumento para a avaliação das FE. Foi possível observar que é comum a aplicação dos instrumentos WCST, Escalas Wechsler e Teste de Stroop em conjunto na mesma avaliação, verificando-se essa combinação em cinco dos artigos analisados (Duchesne et al., 2010; Netto et al., 2011; Pedron et al., 2015; Zartman et al., 2013; Lima et al., 2013). Contudo, a combinação mais frequente ao longo das avaliações é a aplicação em conjunto do teste WCST e das Escalas Wechsler, observada em 11 artigos (Duchesne et al., 2010; Freire et al., 2015; Lima et al., 2013; Lopes et al., 2011; Lopes, 2010; Lopes et al., 2013; Martino et al., 2012; Netto et al., 2011; Pavan et al., 2015; Pedron et al., 2015; Zartman et al., 2013).

Conforme Lopes et al. (2013), as Escalas Wechsler, especificamente a escala WAIS-III, é mundialmente usada para a avaliação de habilidades das FE, a exemplo da flexibilidade cognitiva, estratégias de solução de problemas e memória imediata. Já o Teste WCST é utilizado objetivando avaliar a flexibilidade cognitiva na resolução de problemas, a atenção e o controle de impulsos (Strauss, Sherman, \& Spreen, 2006; Brito et al., 2012). Desse modo, a utilização dos dois instrumentos nos vários estudos pode ser justificada pelo fato de que, em conjunto, o Wisconsin e as Escalas Wechsler avaliam os três componentes ou funções executivas básicas consentidas por diversos autores, que são: a memória de trabalho, a inibição, ou controle inibitório, e a flexibilidade cognitiva (Diamond, 2013; Dias et al., 2015; Ferreira et al., 2015, Miyake et al., 2000).

Tais combinações de baterias e testes podem associar-se ao fato de que o avaliador utiliza-se das baterias e dos testes que, em conjunto, afiram de forma mais completa as $\mathrm{FE}$, isso devido a não existência de um instrumento único que avalie a todos os componentes executivos, exigindo a aplicação de subtestes isolados. Além disso, o uso de baterias flexíveis no contexto da avaliação neuropsicológica decorre, segundo Mattos e Paixão Júnior (2010), da existência de poucos instrumentos normatizados, o que impede o uso de baterias neuropsicológicas fixas que englobem a todos os aspectos que se pretendem avaliar. Destaca-se que nos estudos que fizeram o uso de apenas um instrumento para a avaliação das FE foram observados processos de validação e adaptação do instrumento, ou mesmo a avaliação das propriedades psicométricas e da sensibilidade deste. (Cardoso et al., 2015; Fontoura et al., 2011; Oliveira et al., 2014; Salles et al., 2011; Silva et al., 2015).

No que diz respeito às finalidades às quais destinavam-se às avaliações das FE empreendidas, percebeu-se que algumas pesquisas objetivaram com o uso dos instrumentos comparar grupos clínicos, especialmente comparações entre indivíduos que apresentam algum transtorno e/ou doença e outros sem esses quadros, a exemplo do transtorno do pânico (Bolshaw, Greca, Nardi, Júnior, Fonseca, \& Fernandez, 2011), traumatismo craniano (Fonseca et al., 2012) e acidente cerebrovascular (Morrison et al., 2013).

Além disso, foram encontrados artigos que utilizaram instrumentos objetivando identificar a relação entre FE e determinadas patologias, tais como o Transtorno Obsessivo Compulsivo, a Obesidade, o Déficit de Atenção/Hiperatividade, o Transtorno depressivo, o Transtorno bipolar do humor e a Diabetes (Coelho, Albuquerque, \& Simões, 2013; Duchesne et al., 2010; Hovik et al., 2015; Lopes, 2010; Martino, Strejilevich, \& Manes, 2012; Nunes et al., 2014; Pedron, Ferrão, Gurgel, \& Reppold, 2015). Corroborando com os resultados apresentados, Bolshaw et al. (2011) afirmam que se nota um recente e significativo aumento no número de publicações em busca de um perfil neuropsicológico de quadros psiquiátricos, tendo sido realizados estudos com os mais diversos transtornos mentais e deficiências. No que se refere às FE, suas associações com grupos clínicos ocorrem levando-se em consideração as alterações em algum dos componentes executivos, observadas a partir dos escores nos instrumentos de avaliação.

Quanto às populações investigadas nessas publicações, foi possível observar que a maioria (17 referências) apresenta, enquanto os sujeitos/participantes investigados da pesquisa, indivíduos adultos (acima dos 18 anos de idade), seguido em número de publicações da população idosa, acima de 60 anos de idade (15 referências), de crianças, abaixo de 12 anos de idade (nove referências), e de adolescentes, entre 12 e 17 anos de idade (cinco referências), conforme apresentou a Tabela 1, acima.

Pode-se justificar a escolha preferencial dos pesquisadores pela população adulta na medida em que a maioria dos instrumentos validados e adaptados apresentam tabelas de padronização e normatização para sujeitos adultos, assim sendo, torna-se mais difícil a análise das demais populações. Conforme Pires (2010), a falta de medidas adequadas em termos desenvolvimentais tem dificultado a avaliação clínica das FE. Referindo-se ao contexto da avaliação neuropsicológica pediátrica das FE, Barros e Hazin (2013) afirmam que a maioria dos instrumentos são 
adaptações ou aplicações de medidas inicialmente desenvolvidas para adultos, de modo a tornar a avaliação significativamente mais difícil e menos precisa.

Nos artigos que utilizam instrumentos na avaliação de crianças e adolescentes, observou-se um interesse específico pela relação entre FE e o processo de aprendizagem, assim como pelas dificuldades encontradas neste (Boschloo et al., 2014; Lima et al., 2013; Freire et al., 2015; Pazeto, et al., 2014). Pode-se afirmar que o interesse por essa relação decorre do fato de que se demonstra que as habilidades executivas são preditoras de um bom desempenho na aprendizagem e que o prejuízo em algumas dessas habilidades pode levar a dificuldades de aprendizagem (Corso, Sperb, Jou, \& Salles, 2013). Enquanto isso, nas análises de indivíduos idosos, notou-se o interesse primordial pela avaliação da relação entre FE, demências e doenças crônico-degenerativas, constatando o interesse dos pesquisadores pelo esperado declínio dessas habilidades com o passar dos anos, já que as FE são uma das primeiras habilidades a declinarem no envelhecimento, principalmente no que concerne a flexibilidade cognitiva e a memória de trabalho (Wachholz \& Yassuda, 2011).

Destaca-se que 25 pesquisas têm como público-alvo uma faixa etária especificamente, enquanto que nove analisam duas simultaneamente e, uma pesquisa (Fontoura et al., 2011) leva em consideração as três faixas etárias (adolescentes, adultos e idosos). Tais resultados podem decorrer do fato de existirem poucos instrumentos que avaliem as funções executivas em todas as faixas etárias, assim como das características do desenvolvimento e declínio natural das FE, de modo que devem-se utilizar parâmetros diferentes para avaliar as distintas faixas etárias.

Considerando os dados obtidos a partir da presente revisão, foi possível observar um avanço em relação as revisões anteriores, tendo em vista que foram identificados e discutidos estudos com participantes em diferentes faixas etárias, o que permitiu um panorama geral das publicações na área, a identificação dos instrumentos mais utilizados na avaliação das FE e os possíveis motivos que levam os autores a utilizá-los. Além disso, foram abordados brevemente os parâmetros psicométricos dos instrumentos identificados, permitindo iniciar uma discussão e a reflexão sobre a importância da criação de instrumentos específicos, adaptados e normatizados que sejam precisos e válidos para avaliações clínicas e educacionais.
Destaca-se que são necessárias novas pesquisas de revisão e empíricas que centrem a atenção na população de crianças e adolescentes, assim nos componentes/habilidades executivas, tendo em vista a falta de consenso teórico na área das FE, especialmente no que tange aos seus componentes, e o pequeno número de pesquisas, tanto nacionais quanto internacionais, que investiguem as populações de crianças e adolescentes.

\section{Considerações Finais}

Os resultados apontaram para um aumento no número de publicações empíricas nos últimos três anos, revelando o maior interesse dos pesquisadores pela investigação das funções executivas. No entanto, o número de pesquisas na área ainda é incipiente. Verifica-se a relevância teórica da construção de um consenso a respeito da definição das FE, pois, devido a sua difícil delimitação, conduz-se a distintas medidas de avaliação neuropsicológica, levando ao enfraquecimento dos achados científicos no intuito de alcançar dados normativos para o desenvolvimento típico ou atípico dessas funções.

Nesse ínterim, nota-se que a avaliação das FE é composta por diferentes tipos de instrumentos, processos e técnicas. Observa-se a partir desta revisão que sua mensuração é complexa, sendo realizada a partir do uso conjunto de vários instrumentos neuropsicológicos, tendo em vista ainda não existir um instrumento composto por tarefas que avaliem pelo menos os três componentes executivos básicos, destacados por Miyake et al. (2000).

Em suma, o presente artigo objetivou apresentar um panorama das pesquisas empíricas na área de funções executivas, atentando-se para os desenhos metodológicos utilizados nos artigos, para os instrumentos atualmente mais aplicados, para a frequência de publicações, bem como para as populações mais frequentemente investigadas. Destaca-se que os artigos que não disponibilizaram texto completo e gratuitamente disponível nas referidas bases de dados, escritos em outro idioma (além do português e do inglês) ou resultantes de pesquisas não empíricas foram excluídos da análise, podendo ser incluídos em futuras pesquisas. Ressalta-se que os achados deste estudo visam direcionar e incentivar pesquisas futuras na área de avaliação neuropsicológica das funções executivas, em especial, voltadas para a construção de instrumentos e considerando principalmente as populações infantil e adolescente, que são as atualmente menos exploradas.

\section{Referências}

American Educational Research Association [AERA], American Psychological Association [APA], \& National Council on Measurement in Education [NCME], (2014). Standards for educational and psychological testing. Washington, DC: American Educational Research Association. 
Army Individual Test Battery - AIT. (1944). Manual of directions and scoring. Washington, DC: War Department, Adjutant General's Office.

Balaguero, M. A., Mercé, J. V., Molina, A. G., Tormos, J. M., \& Rovira, T. R. (2016). Balloon Analogue Risk Task to assess decision-making in Acquired Brain Injury. International Journal of Psychological, 9(1), 30-39. Recuperado de http://www.scielo.org.co/scielo.php?script=sci_ arttext\&pid=S2011-20842016000100004\&lng=en\&tlng=en

Barros, P. M., \& Hazin, I. (2013). Avaliação das Funções Executivas na Infância: Revisão dos Conceitos e Instrumentos. Psicologia em Pesquisa, 7(1), 13-22. doi: 10.5327/Z1982-1247201300010003

Bolshaw, M., Greca, D. V., Nardi, A E., Júnior, E. C., Fonseca, R. P., \& Fernandez, J. L. (2011). Funções cognitivas no transtorno do pânico: Um estudo comparativo com controles saudáveis. PSICO: 42(1), 87-97. Recuperado de http://repositorio.pucrs.br/dspace/ bitstream/10923/9333/2/Funcoes_cognitivas_no transtorno_do_panico_um_estudo_comparativo_com_controles_saudaveis.pdf

Borsa, J. C., Damásio, B. F., \& Bandēira, D. R. (2012). Adaptação e validaçãāo de instrumentos psicológicos entre culturas: Algumas considerações. Paidéia (Ribeirão Preto), 22(53), 423-432.doi: 10.1590/S0103-863X2012000300014

Boschloo, A., Krabbendam, L., Aben, A., Groot, R., \& Jolles, J. (2014). Sorting Test, Tower Test, and BRIEF-SR do not predict school performance of healthy adolescents in preuniversity education. Frontiers in Psychology, 287(5), 1-8. Recuperado de file:///C:/Users/ Masterlar/Desktop/fpsyg-05-00287.pdf

Brito, I. L., Areosa, S. C., Lopes, R. M. F., \& Argimon, I. I. L. (2012). Avaliação das funções executivas em idosos acometidos por doenças crônico-degenerativas. Cuadernos de neuropsicología, 6(1), 46-62. doi: 10.7714/cnps/6.1.203

Cardoso, C. O., Zimmermann, N., Paraná, C. B., Gindri, G., Pereira, A. P. A., \& Fonseca, R. P. (2015). Brazilian adaptation of the Hotel Task: A tool for the ecological assessment of executive functions. Dement Neuropsychol., 9(2),156-164. doi: 10.1590/198057642015DN92000010

Carvalho, J. C. N., Cardoso, C. de O., Cotrena, C., Bakos, D. S., Kristensen, C., \& Fonseca R. P. (2012). Tomada de decisão e outras funções executivas: Um estudo correlacional. Ciências \& Cognição, 17(1), 94-104. Recuperado de http://pepsic.bvsalud.org/scielo. php?script $=$ sci_arttext\&pid $=$ S1806-58212012000100008\&lng $=$ pt\&tlng $=$ pt

Coelho, S., Albuquerque, C. P., \& Simões, M. R. (2013). Distúrbio específico de linguagem: Caracterização neuropsicológica. Paidéia (Ribeirão Preto), 23(54), 31-41. doi: 10.1590/1982-43272354201305

Corso, H. V., Sperb, T. M., Jou, G. I., \& Salles, J. F. (2013). Metacognição e funções executivas: Relações entre os conceitos e implicações para a aprendizagem. Psicologia: Teoria e Pesquisa, 29(1), 21-29. Recuperado de http://www.scielo.br/pdf/ptp/v29n1/04.pdf

Cunha, J. A. (2005). Teste Wisconsin de Classificação de Cartas - Adaptação e padronização Brasileira. São Paulo: Casa do Psicólogo.

Diamond, A. (2013). Executive functions. Annual Review of Psychology, 64, 135-168. doi: 10.1146/annurev-psych-113011-143750

Dias, N. M., Gomes, C. M. A., Reppold, C. T., Fioravanti-Bastos, A. C. M., Pires, E. U., Carreiro, L. R. R., \& Seabra, A. G. (2015). Investigação da estrutura e composição das funções executivas: Análise de modelos teóricos. Psicologia: teoria e prática, 17(2), 140-152. Recuperado de http://pepsic.bvsalud.org/scielo.php?script=sci_arttext\&pid=S1516-36872015000200011\&lng=pt\&tlng=pt

Duchesne, M., Mattos, P., Appolinário, J. C., Freitas, S. R., Coutinho, G., Santos, C., \& Coutinho, W. (2010). Assessment of executive functions in obese individuals with binge eating disorder. Revista Brasileira de Psiquiatria, 32(4), 381-388. doi: 10.1590/S151644462010000400011

Faria, C. A, Alves, H. V. D, \& Charchat-Fichman, H. (2015). The most frequently used tests for assessing executive functions in aging. Dementia \& Neuropsychologia, 9(2), 149-155. doi: 10.1590/1980-57642015DN92000009

Ferreira, L. de O., Zanini, D. S., \& Seabra, A. G. (2015). Executive functions: Influence of sex, age and its relationship with intelligence. Paidéia (Ribeirão Preto), 25(62), 383-391. doi: 10.1590/1982-43272562201512

Fonseca, R. P., Zimmermann, N., Cotrena, C., Cardoso, C., Kristensen, C. H., \& Grassi-Oliveira, R. (2012). Neuropsychological assessment of executive functions in traumatic brain injury: Hot and cold components. Psychology E Neuroscience, 5(2), 183-190. doi: 10.3922/j. psns.2012.2.08

Fontoura, D. R., Rodrigues, J. C., Fonseca, R. P., Parente, M. A. M. P., \& Salles, J. F. (2011). Adaptação do Instrumento de Avaliação Neuropsicológica Breve NEUPSILIN para avaliar pacientes com afasia expressiva: NEUPSILIN-Af. Ciências \& Cognição, 16(3), 78-94. Recuperado de http://pepsic.bvsalud.org/scielo.php?script=sci_arttext\&pid=S1806-58212011000300008\&lng=pt\&tlng=pt

Freire, T., Gonçalves, T. S., Moretti, C. N., Tabaquim, M. L. M., \& Crenitte, P. A. P. (2015) Desempenho nas habilidades subjacentes a aprendizagem em um caso de Sindrome Velocardio facial. Distúrbios da Comunicação, 27(2), 237-247. Recuperado de http://revistas. pucsp.br/index.php/dic/article/view/20150

Galvão, T. F., Pansani, T. S. A., \&Harrad, D. (2015). Principais itens para relatar revisões sistemáticas e meta-análises: A recomendação PRISMA. Epidemiologia e Serviços de Saúde, 24(2), 335-342. Recuperado de http://www.scielo.br/scielo.php?script=sci_arttext\&pid=S2237$96222015000200335 \& \operatorname{lng}=$ en\&nrm $=$ iso

Goode, W., \& Hatt, P. (1973). Métodos em pesquisa social. São Paulo, SP: Nacional.

Heaton, R. K., Chelune, G. J., Talley, J. L., Kay, G. G., \& Curtiss, G. (2004). Teste Wisconsin de Classificação de Cartas. São Paulo: Casa do Psicólogo

Hovik, K. T., Plessen, K. J., Cavanna, A. E., Skogli, E. W., Andersen, P. N., \& Øie, M. (2015). Cognition, Emotion and Behavior in Children with Tourette's Syndrome and Children with ADHD - Combined Subtype - A Two-Year Follow-Up Study. PLOS ONE, 10(12), e0144874 doi:10.1371/journal.pone.0144874

Ihnen, J., Antivilo,A., Muñhoz-Neira, C., \& Slachevsky, A (2013). Chilean version of the INECO Frontal Screening (IFS-Ch): psychometric properties and diagnostic accuracy. Dementia Neuropsychologia, 7(1), 40-47. Recuperado de http://www.demneuropsy.com.br/detalhe_ artigo.asp?id $=373$

Kluwe-Schavion, B., Viola, T. W., \& Grassi-Oliveira, R. (2012). Modelos teóricos sobre constructo único ou múltiplos processos das funções executivas. Revista Neuropsicologia Latinoamericana, 4(2), 29-34. Recuperado de https://www.neuropsicolatina.org/index.php/ Neuropsicologia_Latinoamericana/article/view/106/80

Lima, R. F., Azoni, C. A. S., \& Ciasca, S. M. (2013). Atenção e funções executivas em crianças com dislexia do desenvolvimento. Psicologia em Pesquisa, 7(2), 208-219. Recuperado de http://pepsic.bvsalud.org/pdf/psipesq/v7n2/09.pdf

Lopes, R. M. F. (2010). Desempenho cognitivo de idosos com diabetes Mellittus tipo 2 no teste Wisconsin de classificação de cartas (WCST). PUC/RS/Psicologia, 9(3), 697-713. Recuperado de http://www.dominiopublico.gov.br/pesquisa/DetalheObraForm. do? select_action $=\&$ co_obra $=135756$ 
Lopes, R. M. F., Nascimento, R. F. L., Esteves, C. S., Iatchac, F.O., \& Lima, I. I. (2011). Cognição e Diabetes Mellitus tipo 2 em idosos. Ciências \& Cognição, 16(3), 95-108. Recuperado de http://pesquisa.bvs.br/brasil/resource/pt/psi-58791

Lopes, R. M. F., Nascimento, R. F. L., Esteves, C. S., Terroso, L. B., \& Argimon, I.I. de L. (2013). Funções executivas de idosos com depressão: Um estudo comparativo. Cuadernos de Neuropsicología, 7(2), 72-86. doi: 10.7714/cnps/7.2.204

Luecht, R. M., \& G. Sireci, S. G. (2011). A Review of Models for Computer-Based Testing. The College Board, College Board Research Reports.

Magila, M. C., \& Caramelli, P. (2000). Funções executivas no idoso. Em O. V. Forlenza. \& P. Caramelli (Orgs.) Neuropsiquiatria geriátrica. (pp. 517-526). São Paulo: Atheneu.

Martino, D. J., Strejilevich, S. A., \& Manes, F. (2012). Neurocognitive functioning in early-onset and late-onset older patients with euthymic bipolar disorder. International Journal of Geriatrc Psychiatry, 28(2), 142-148. doi: 10.1002/gps.3801

Mata, F., Sallum, I., Moraes, P. H. P., Miranda, D. M., \& Malloy-Diniz, L. F. (2013). Development of a computerised version of the Children's Gambling Task for the evaluation of affective decision-making in Brazilian preschool children. Estudos de Psicologia,18(1), 151-157. Recuperado de http://www.scielo.br/pdf/epsic/v18n1/24.pdf

Mattos, P., \& Paixão Júnior, C. M. (2010). Avaliação cognitiva de idosos: Envelhecimento e comprometimento cognitivo leve. Em L.F. Malloy-Diniz, D. Fuentes, P. Mattos, N. Abreu (Eds.). Avaliação Neuropsicológica (pp. 247-253). Porto Alegre: Artmed.

Miyake, A., Friedman, N. P., Emerson, M. J., WItzki, A. H., Howerter, A., \& Wager, T. D. (2000). The unity and diversity of executive functions and their contributions to complex "frontal lobe" tasks: A latent variable analysis. Cognitive Psychology, 41(1), 49-100. doi: 10.1006/cogp.1999.0734

Möller, M. C., Boussard, C., Oldenburg, C., \& Bartfai, A. (2014). An investigation of attention, executive, and psychomotor aspects of cognitive fatigability. Journal of Clinicanl and Experimental Neuropsychology, 36(7), 716-29. doi: 10.1080/13803395.2014.933779

Morrison, M. T., Giles, G. M., Ryan, J. D., Baum, C. M., Dromerick, A. W., Polatajko, H. J., \& Edwards, D. F. (2013). Multiple Errands Test - Revised (MET-R): A Performance-Based Measure of Executive Functionin People With Mild Cerebrovascular Accident. The American Journal of Occupational Therapy, 67(4), 460-468. Recuperado de https://www.ncbi.nlm.nih.gov/pmc/articles/PMC5360199/pdf/460.pdf

Netto, T. M., Greca, D. V., Ferracini, R., Pereira, D. B., Bizzo, B., Doring, T., Kubo, T., ..., \& Gasparetto, E. L. (2011). Correlação entre espessura cortical frontal e desempenho de funções executivas em pacientes com infecção pelo vírus da imunodeficiência humana. Radiologia Brasileira, 44(1), 7-12. doi: 10.1590/S0100-39842011000100006

Nunes, D., Monteiro, L., \& Lopes, E. (2014). INECO frontal screening: Um instrumento para avaliar as funções executivas na depressão. Psicologia Clínica, 26(2), 177-196. Recuperado de http://www.scielo.br/scielo.php?script=sci_arttext\&pid=S0103$56652014000200011 \& \operatorname{lng}=\mathrm{en} \& \mathrm{t} \operatorname{lng}=\mathrm{pt}$

Oliveira, A. P. A., \& Nascimento, E. do. (2014). Construção de uma Escala para Avaliação do Planejamento Cognitivo. Psicologia: Reflexão e Crítica, 27(2), 209-218. doi: 10.1590/1678-7153.201427201

Oliveira, C. R. Pedron, A. C., Gurge, L. G., Reppold, C. T., \& Fonseca, R.P. (2012). Executive functions and sustained attention: Comparison between age groups of 19-39 and 40-59 years old. Dementia and Neuropsychology, 6(1), 29-34. doi: 10.1590/S198057642012DN06010005

Ottati, F., \& Noronha, A. P. P. (2003). Parâmetros psicométricos de instrumentos de interesse profissional. Estudos e Pesquisas em Psicologia, 3(2), 37-50. Recuperado de http://pepsic.bvsalud.org/scielo.php?script=sci_arttext\&pid=S1808-42812003000200003\&lng $=$ pt\&tl $\mathrm{ng}=\mathrm{pt}$

Pasquali, L. (Ed.). (2001). Técnicas de exame psicológico - TEP. Manual. (Vol. I). São Paulo: Casa do Psicólogo; Brasília: Conselho Federal de Psicologia.

Pavan, L. S., Casarin, F. S., Pagliarin, K. C., \& Fonseca, R. P. (2015). Avaliação neuropsicológica no Acidente Vascular Cerebral: um estudo de caso. Distúrbios da Comunicação, 27(4), 831-839. Recuperado de http://revistas.pucsp.br/index.php/dic/article/viewFile/22665/18815

Pazeto, T. C. B., Seabra, A. G., \& Dias, N. M. (2014). Executive functions, oral language and writing in preschool children: Development and correlations. Paidéia (Ribeirão Preto), 24(58), 213-222.doi:10.1590/1982-43272458201409

Pedron, A. C., Ferrão, Y. A., Gurgel, L. G., \&Reppold, C. T. (2015). Relations between executive functions and different symptomatic dimensions in obsessive compulsive disorder. Paidéia (Ribeirão Preto), 25(61), 229-239. doi: 10.1590/1982-43272561201511

Pires, E. U. (2010). Ontogênese das funções cognitivas: Uma abordagem neuropsicológica (Dissertação de mestrado). Programa de Pós-Graduação em Psicologia Clínica, Centro de Teologia e Ciências Humanas, Pontifícia Universidade Católica do Rio de Janeiro. Rio de Janeiro, Brasil.

Ramos, A. A., \& Hamdan, A. C. (2016). O crescimento da avaliação neuropsicológica no Brasil: uma revisão sistemática. Psicologia: Ciência e Profissão, 36(2), 471-485. doi: 10.1590/1982-3703001792013

Ritter, B. C., Perrig, W., Steinlin, M., \& Everts, R. (2013). Cognitive and behavioral aspects of executive functions in children born very preterm. Child Neuropsychology, 20(2), 129-44. doi: 10.1080/09297049.2013.773968.

Salles, J. F., Fonseca, R. P., Cruz-Rodrigues, C., Mello, C. B., Barbosa, T., \& Miranda, M. C. (2011). Desenvolvimento do Instrumento de Avaliação Neuropsicológica Breve Infantil NEUPSILIN-INF. Psico-USF, 16(3), 297-305. doi: 10.1590/S1413-82712011000300006

Silva, M., Leite, M. F. B., Zongo, H. M, Madureira, C., \& Peixoto, B. (2015). Angolan Version of Ineco Frontal Screening: psychometric indicators and discriminative capacity in post-traumatic stress disorder. Journal of Clinical and Biomedical Sciences, 3535(1413),141-148. doi: 10.43222357-9730.56913

Silva-Filho, J. H., Pasian, S. R., \& Barboza, L. L. (2013). Potencial informativo e desafios técnicos do Teste Wisconsin de classificação de cartas. Revista da SPAGESP, 14(2), 102-113. Recuperado de http://pepsic.bvsalud.org/pdf/rspagesp/v14n2/v14n2a08.pdf

Souza, R. O., Moll, J., Ignácio, F. A., \& Moll, F.T. (2008). Cognição e funções executivas. Em Lent, R. (Org.) Neurociência da mente e do comportamento (pp. 303-326) Rio de Janeiro: Guanabara Koogan.

Spreen, O., \& Strauss, E. (1991). A Compendium of Neuropsychological Tests - Administration, Norms and Commentary. New York; Oxford: Oxford University Press.

Strauss, E., Sherman, E. M. S., \& Spreen, O. (2006). A compendium of neuropsychological tests: administration, norms, and commentary. Oxford: Oxford University Press.

Verdejo-Garcia, A., \& Bechara, A. (2010). Neuropsicologia de las funciones ejecutivas. Psicothema, 22(2), 227-235., Recuperado de https:// www.redalyc.org/articulo.oa?id $=72712496009$ 
Wachholz, T. B. O., \& Yassuda, M. Sá (2011). The interpretation of proverbs by elderly with high, medium and low educational level: Abstract reasoning as an aspect of executive functions. Dementia \& Neuropsychologia, 5(1), 31-37. doi: 10.1590/S1980-57642011DN05010006)

Zartman A. L., Hilsabeck, R. C., Guarnaccia, C.A., \& Houtz A. (2013). The Pillbox Test: An ecological measure of executive functioning and estimate of medication management abilities. Archives of Clinical Neuropsychology, 28(4), 307-319. doi: 10.1093/arclin/act014

Alanny Nunes de Santana é graduada em Psicologia pela Universidade Federal de Campina Grande, UFCG, PB, Brasil e mestranda em Psicologia Cognitiva pela Universidade Federal de Pernambuco, UFPE, PE, Brasil.

Monilly Ramos Araujo Melo é doutora em Psicologia Cognitiva e docente do curso de Psicologia da Universidade Federal de Campina Grande, UFCG, PB, Brasil.

Carla Alexandra da Silva Moita Minervino é doutora em Ciências da Saúde pela Universidade Federal da Paraíba e docente na Universidade Federal da Paraíba, UFPB, PB, Brasil. 